\title{
Simulation of unsteady muzzle flow of a small-caliber gun
}

\author{
Y. Dayan \& D. Touati \\ Department of Computational Mechanics \& Ballistics, \\ IMI, Ammunition Group, Israel
}

\begin{abstract}
The present paper presents a simulation of a firing process of a $7.62 \mathrm{~mm}$ bullet and muzzle flow out of a gun barrel. The calculation is made in two stages. First, an internal ballistics (IB) simulation via the IBHVG2 software package, is performed in order to obtain bullet travel dependent breech pressure and temperature. Following this, a simulation of unsteady muzzle flow is made, beginning at the start of bullet motion and ending one meter beyond the muzzle exit point. The second stage calculation is carried out via the CFD-FASTRAN finite volume solver package. The movement of the bullet is simulated by a chimera overset meshing technique. In general, there exists very good agreement between the computed IB and the measured muzzle velocity and pressures, and between the CFD precursors flow field, main propellant flow field calculations and the experimental shadowgraph results.

Keywords: blast, CFD-FASTRAN, chimera overset grid, first precursor, second precursor, mach disk, main propellant flow.
\end{abstract}

\section{Introduction}

Gun muzzle signature, blast and flash phenomena are of practical importance to the gun designer, especially their influence on firing accuracy. Overpressures and intense radiation affect the gun crew and surroundings and can be minimized by muzzle attachments that reduce the momentum of the exit flow $[1,2]$.

One of the challenges is to simulate the blast process numerically. The simulation results of the precursor flow field and main flow field phenomena help us to better understand the forces and present during these processes (jump phenomena). Another important aspect involves finding the forces acting on the 
bullet for asymmetric flows and their influence on the subsequent bullet's trajectory once it exits the muzzle [7-9].

Considerable progress has been made in the area of unsteady flow analyses of multiple moving bodies with the aid of CFD. One of the approaches currently used to solve the unsteady muzzle flow of a bullet (fired from a gun barrel) is to generate a grid for the bullet by a chimera/overset methodology [3], and the latter moves on the gun barrel's fixed grid via a six-degree-of-freedom model based on the theory of Etkin [4].

Figure 1 depicts the firing process of a $7.62 \mathrm{~mm}$ bullet and muzzle flow out of a gun barrel.
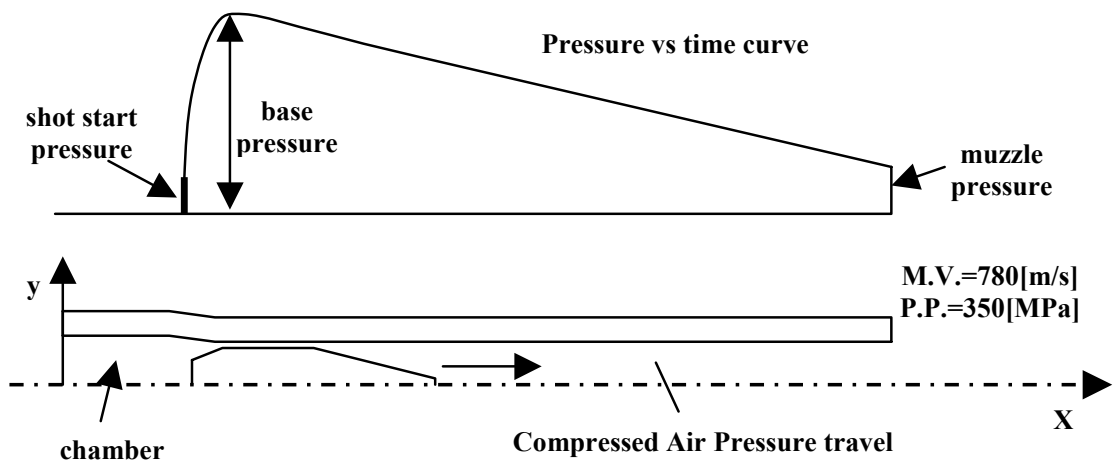

Figure 1: Pressure vs axial distance from the breech along gun tube for axisymmetric bullet.

\section{Process description}

One or two precursor flow fields and the main propellant gas plume are formed about the muzzle of the gun during its firing. These two or three impulsive jet flows that subsequently exit the muzzle of the gun interact with each other. The precursor flow fields are rapidly overtaken and destroyed by the main propellant flow.

The precursor flow is generated inside the gun tube due to the compression waves produced by the accelerating projectile, leaked propellant gas-particle flow. These waves rapidly coalesce into a steep precursor shock front, and then exit at the muzzle of the gun tube, expanding into the ambient atmosphere. A later stage of the precursor flow development is shown in the schematic drawing of figure 2. The air-air or gas-air interface ends laterally in a vortex due to the shear forces acting around the edge of the jet flow. The Mach disk forms and decelerates from supersonic to subsonic flow velocity. The gas-air interface in the axial direction takes the form of a distinct cap surface. The barrel shock develops undisturbed and terminates in the triple point from which the reflected shock and Mach disk originate. 
After the bullet exits the gun barrel, the flow of the main propellant gases begins. The propellant gases exit the barrel at a pressure ratio of more than 500 . Because the pressure ratio is much higher, the precursor blast wave is quickly overtaken.

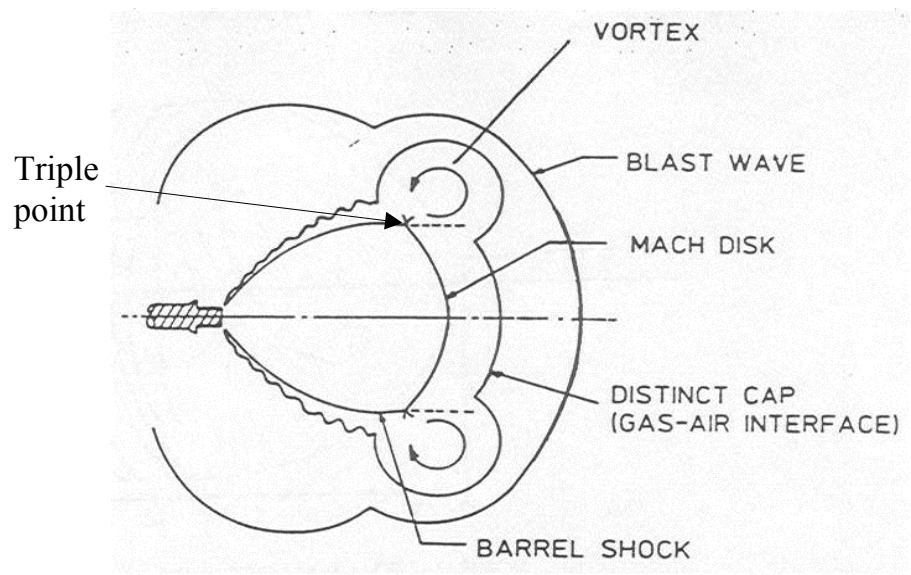

Figure 2: $\quad$ Later stage of precursor flow field (ref. [1]).

\section{Numerical methods approach}

The solution procedure involves solving the unsteady, conservative, NavierStokes equations. The numerical scheme employed is a finite-volume, Riemann solver with an upwind difference method for computed spatial derivatives. Fluxes are computed using Van leer's flux vector splitting scheme with second order accuracy. A fully implicit time integration scheme is employed to advance the solution in time, ensuring global time accuracy with a time step of 1.0e-007 sec and with 250 sub-iterations between each time step. The solution also includes a k- $\varepsilon$ turbulence model with a wall function formulation.

The moving body (the bullet) problem solution involves solving the rigid body equations of motion accounting for the hot gas forces behind the bullet. The bullet is modelled by a Chimera methodology with a multi zone overlapped grid approach. It provides the flexibility of modelling geometries with multi domain grids in which different zones are allowed to overlap each other in an arbitrary manner. In the case of complex problems that involve multiple moving bodies, this methodology facilitates independent generation of grids, which could be tied together with other grids in the domain.

The major grid (gun tube) consisted of 160,000 grids points, while the minor grids (bullet) consisted of 20,000 points. The barrel length is $0.4 \mathrm{~m}$. 


\subsection{CFD-FASTRAN flow solver}

The following Navier-Stokes equations are solved using the finite volume method [3]:

$$
\int \frac{\partial \vec{Q}}{\partial t} d V+\int \nabla \cdot \vec{F}^{n+1} d V=0
$$

where the vector $Q=(\rho, \rho u, \rho v, \rho w, E)$; the tensor $\vec{F}=(F, G, H)$ and where $F$ and $G$ are the inviscid and viscous flux vectors, respectively, $H$ is the vector of source terms, $V$ is the cell volume, and $A$ is the surface area of the cell face.

\subsection{Equation of chimera grid motion}

The 6DOF motion model requires additional information to determine how the body moves, based on physical properties and forces [5]. This is achieved through the general equations of unsteady motion. The equations of motion for a rigid body with constant mass and mass moments of inertia as given by [4] are:

$$
\begin{gathered}
\vec{F}=m \frac{d \vec{v}}{d t} \\
\vec{M}=\frac{\partial \vec{h}}{\partial t}+\vec{\omega} \times \vec{h}
\end{gathered}
$$

where

$\vec{F}=$ the resultant force vector of all forces

$\vec{M}=$ the moment vector about the body's centre of gravity

$m=$ the body mass

$\vec{v}=$ linear velocity vector of the centre of gravity

$\vec{h}=$ angular momentum

$\vec{\omega}=$ the angular velocity vector about the body's centre of gravity.

\section{Results}

The gun barrel selected for this CFD validation is a $7.62 \mathrm{~mm}$ with a $0.4 \mathrm{~m}$ barrel length. This configuration is characterized by two precursor flow fields and the main propellant flow field. Figures 3 through 6, present shadowgraphs (ref. [1]) and simulated density contour results starting from $370 \mu \mathrm{sec}$ before bullet ejection until $400 \mu \mathrm{sec}$ after the bullet exits the barrel.

In figure $3,(370 \mu \mathrm{sec}$ prior to bullet ejection) the outer spherical shock of the first precursor has developed (1). The outer blast wave has an identical size and shape to the CFD-FASTRAN simulation. In addition the vortex which is beginning to form, is also of similar size and shape as compared to the shadowgraph image. From the simulation, at this point in time, the bullet's position is $0.05 \mathrm{~m}$ from the muzzle. 
In figure 4 (at $40 \mu \mathrm{sec}$ prior to bullet ejection) the second precursor shock (2) appears. The simulated blast wave for the second precursor (from the CFDFASTRAN simulation) is similar to the shadowgraph image. The Mach disk from the first precursor is clearly visible in the simulation but barely distinguishable in the shadowgraph. (it can barely be seen inside the plume turbulence). At this point in time, the bullet's position is $0.007 \mathrm{~m}$ from the muzzle.

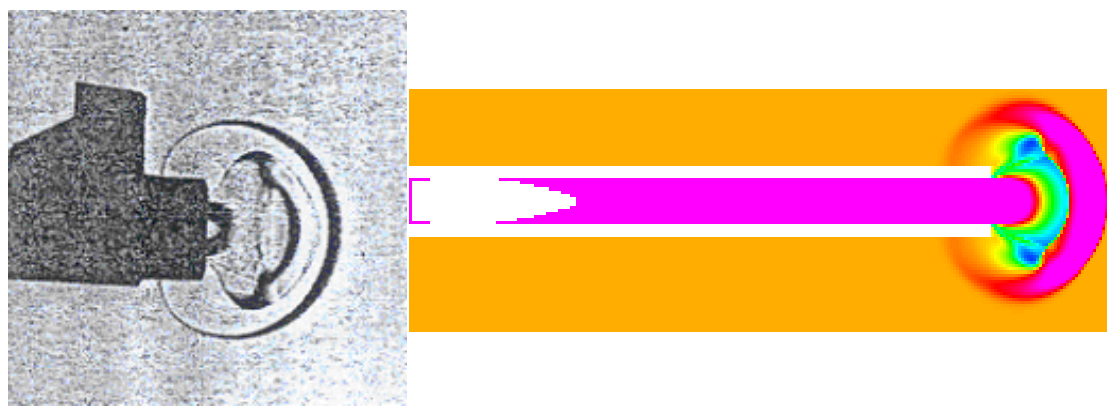

Figure 3: Shadowgraph (from ref. [1]) result versus simulation result of the first precursor flow field $(370 \mu \mathrm{sec}$ before bullet ejection).
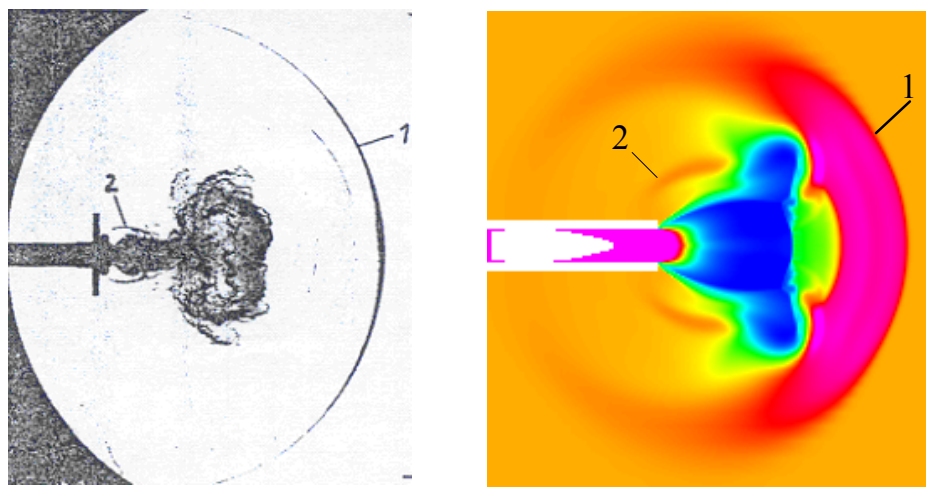

Figure 4: Shadowgraph (from ref. [1]) result versus simulation result of the first (1) and second (2) precursor flow fields $(40 \mu \mathrm{sec}$ before bullet ejection).

At $60 \mu \mathrm{sec}$ after bullet ejection (see figure 5), the main propellant flow field (3) has developed. The beginning of the distortion of the first blast wave can be seen more clearly from the CFD-FASTRAN results. The blast wave of the propellant flow engulfs the base of the bullet, and a nearly vertical shock front is formed. The propellant gases ejected from the muzzle are supersonic, and they expand into the ambient region of relatively low gas pressure and low gas density. 
At $400 \mu \mathrm{sec}$ after bullet ejection (figure 6), the propellant flow (3) starts to engulf the blast wave (1) of the first precursor flow and the bullet.
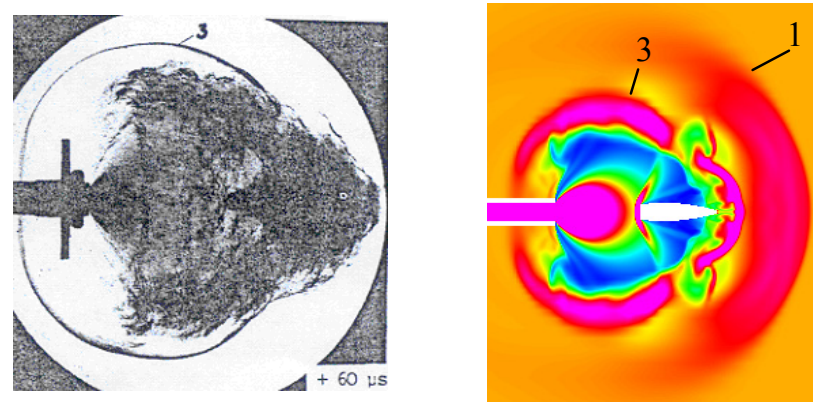

Figure 5: Shadowgraph (from ref. [1]) result versus simulation result of the first precursor flow field (1) and blast wave of main propellant flow (3) $(60 \mu \mathrm{sec}$ after bullet ejection).
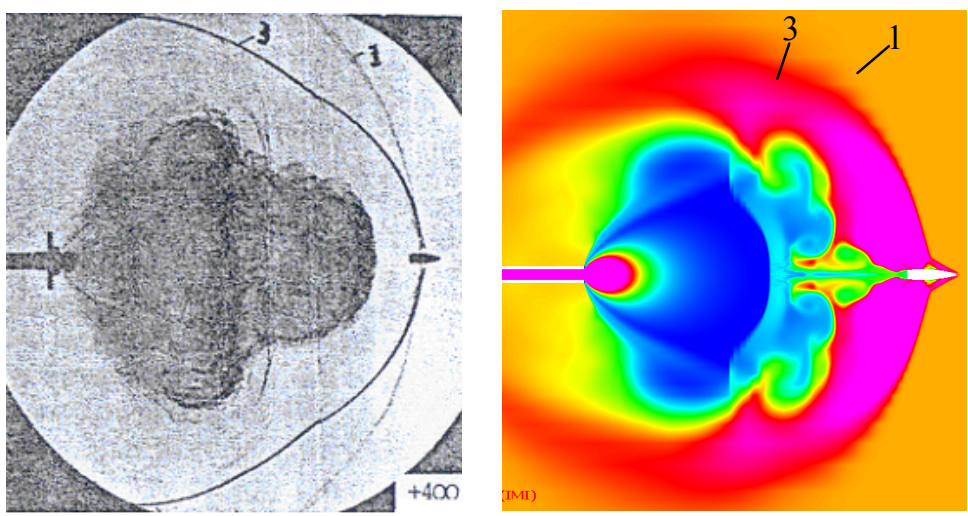

Figure 6: Shadowgraph (from ref. [1]) result versus simulation result of the first precursor flow field (1) and blast wave of main propellant flow (3) $(400 \mu \mathrm{sec}$ after bullet ejection).

\section{Conclusions}

Several conclusions can be drawn from the CFD simulation of gun muzzle blast for a small calibre gun. These include:

- The chimera/overset grid methodology provides accurate meshing models for gun muzzle and bullet motion simulations

- $\quad$ The $7.62 \mathrm{~mm}$ precursor flow fields and the main propellant flow results correspond closely to shadowgraph results 
The precursor flow fields and main propellant flow field results are very sensitive to:

- Local ambient conditions $\left(\mathrm{p}_{\infty}, \rho_{\infty}\right)$

- Shape of the gun barrel and the bullet

- Internal ballistic calculation for peak pressure (P.P), muzzle pressure (M.P.) and muzzle velocity (M.V.)

\section{Acknowledgement}

We wish to thank Mr. Aron Pila of the IMI Ammunition Group's Central Laboratory, for his assistance in editing this paper.

\section{References}

[1] N. Klingenberg and J. M. Heimerl, Phenomenology of Gun Muzzle Flow, In: GUN Muzzle Blast and Flash, AIAA, Vol. 139, pp 107-166.

[2] N. Klingenberg and J. M. Heimerl, Blast Wave Research, In: GUN Muzzle Blast and Flash, AIAA, Vol. 139, pp 167-193.

[3] CFD Research Corporation, CFD-FASTRAN User's Manual; Theory Manual, 2002.

[4] Etkin, B., Dynamics of Flight-Stability and Control, Second Edition, John Wiley \& Sons, Inc., New-York, 1982.

[5] Z. J. Wang, V. Parthasararathy, and N. Hariharan, A Fully Automated Chimera Methodology for Multiple Moving Body Problems, $36^{\text {th }}$ Aerospace Sciences Meeting \& Exhibit, January 12-15, 1998.

[6] A. H. Klaus and T. C. Steve, Computational Fluid Dynamics, A Publication of engineering Education system, ISBN 0-9623731-3-3,Vol 1-3, August 2000.

[7] R. Cayzac, E. Carette, T. Alziary de Roquefort, Intermediate Ballistics Unsteady Sabot Separation: First Computational and Validations, $19^{\text {th }}$ International Symposium of Ballistics, Switzerland, 7-11 May 2001.

[8] A. B. Crowley, and J. Szmelter, Computational of Muzzle Flow Fields Using Unstructured Meshes, $19^{\text {th }}$ International Symposium of Ballistics, Switzerland, 7-11 May 2001.

[9] E. M. Schmidt, Wind Tunnel Measurements of Sabot Discard Aerodynamics, Technical Report ARBRL-TR-02246, July 1980. 